\title{
A Robust Pre-Processing Algorithm for Iris Segmentation From Low Contrast Eye Images
}

\author{
Badejo, J.A. ${ }^{a}$, Atayero, A.A. ${ }^{a}$, Ibiyemi, T.S. ${ }^{b}$ \\ ${ }^{a}$ Department of Electrical and Information Engineering, Covenant University, Ota, Nigeria \\ ${ }^{b}$ Department of Electrical Engineering, University of Ilorin, Ilorin, Nigeria \\ \{joke.badejo, atayero\}@,covenantuniversity.edu.ng,ibiyemits@yahoo.com
}

\begin{abstract}
Iris recognition systems offer highly accurate personal identification both on small and very-large scale systems needed in government, forensic and commercial applications. The automatic segmentation of a noise-free iris region is imperative for optimal performance of the system. However, image characteristics such as brightness and contrast, the differing levels of pigmentation, occlusion by eyelashes and/or eyelids, coupled with varying sensor and environmental conditions, makes iris segmentation a huge and difficult task. This paper proposes an image pre-processing algorithm for robust iris segmentation of low contrast images, aimed at reducing mis-localization errors of basic curve-fitting algorithms. Similar to face detection, the algorithm performs iris detection with a k-NN classifier trained with features extracted by a rotation-invariant texture descriptor based on the co-occurrence of local binary patterns. The integration of the proposed algorithm into an existing open-source iris segmentation module offered a $40 \%$ improvement in execution time; a segmentation accuracy of $92 \%$ was also recorded over 1,898 low contrast eye images acquired from African subjects. The low contrast eye images were acquired to support diversity in iris recognition.
\end{abstract}

Keywords-Iris Detection; Iris Dataset; Iris Segmentation; Pattern Recognition; Local Binary Patterns; Texture Descriptor. 